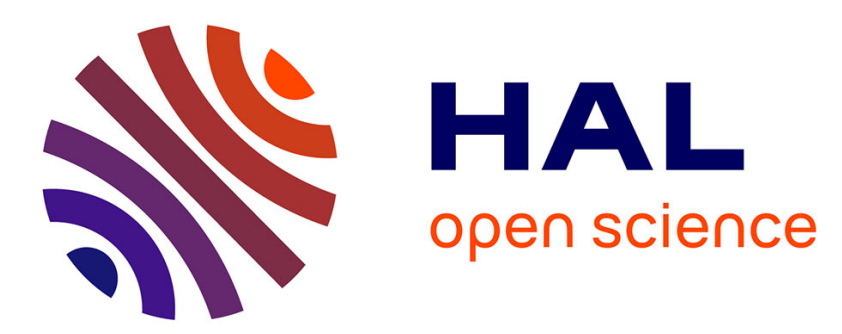

\title{
Mise en évidence de l'instabilité des modèles de réapprovisionnement continu par une analyse dynamique des systèmes logistiques \\ Daniel Thiel
}

\section{- To cite this version:}

Daniel Thiel. Mise en évidence de l'instabilité des modèles de réapprovisionnement continu par une analyse dynamique des systèmes logistiques. Economie Rurale, 1998, 245 (245-246), pp.56-68. 10.3406/ecoru.1998.5017 . hal-02158955

\section{HAL Id: hal-02158955 \\ https://hal.science/hal-02158955}

Submitted on 14 Apr 2021

HAL is a multi-disciplinary open access archive for the deposit and dissemination of scientific research documents, whether they are published or not. The documents may come from teaching and research institutions in France or abroad, or from public or private research centers.
L'archive ouverte pluridisciplinaire HAL, est destinée au dépôt et à la diffusion de documents scientifiques de niveau recherche, publiés ou non, émanant des établissements d'enseignement et de recherche français ou étrangers, des laboratoires publics ou privés. 


\section{Mise en évidence de l'instabilité des modèles de} réapprovisionnement continu par une analyse dynamique des systèmes logistiques

Mr Daniel Thiel

\section{Citer ce document / Cite this document :}

Thiel Daniel. Mise en évidence de l'instabilité des modèles de réapprovisionnement continu par une analyse dynamique des systèmes logistiques. In: Économie rurale. N²45-246, 1998. La grande distribution alimentaire. pp. 56-68;

doi : https://doi.org/10.3406/ecoru.1998.5017

https://www.persee.fr/doc/ecoru_0013-0559_1998_num_245_1_5017

Fichier pdf généré le 26/03/2019 


\title{
Résumé
}

Cet article se propose d'analyser la dynamique des échanges entre distributeurs et producteurs à partir du nouveau paradigme qu'est l'Efficient Consumer Response (ECR). Plus précisément, différents modes de réapprovisionnement continu des magasins de détail et des entrepôts des distributeurs ont été comparés. Des modèles génériques représentant trois schémas d'organisation logistique ont été simulés. La modélisation s'est fondée sur le paradigme de la dynamique des systèmes de Forrester tout en s'appuyant sur une approche empirique des relations industrie-commerce et sur des publications portant sur l'ECR. Une synthèse finale des résultats de simulation comportementale de ces trois modèles est présentée dans l'objectif de saisir l'intérêt commun qu'ont fournisseurs et distributeurs à essayer de comprendre comment réagir rapidement sur la totalité du pipeline logistique.

\begin{abstract}
This paper consists in analysing the dynamics of the exchanges between the distributors and the producers basing the ECR new paradigm. More precisely, different methods of continuous replenishments of shops and distributors warehouses has been compared. Generic models which represent these three logistic organisation diagrams have been implemented. The modelling phase based on Forrester's system dynamics paradigm, used an empirical approach of the relationships between industry and commerce and was based on the papers talking about ECR. Finally, we summarise the major simulation of the three models results in objective to try to improve the understanding of the common politics of the distributors and producers, which rapidly permits to react upon the supply chain.
\end{abstract}




\section{Positionnement de la recherche}

\section{Les origines, les limites et les enjeux des concepts d'organisation logistique}

De nombreux concepts de pilotage des systèmes logistiques ont été construits en se fondant sur une pragmatique professionnelle. C'est le cas du MRP2 (Manufacturing Resources Planning, Wight 1974, 1984), du JIT (Just-inTime ; Shingo 1983; Sekine 1983), ou encore plus récemment de l'ECR (Efficient Consumer Response ; FMI 1993, 1994a-b-c ; GENCOD, 1994, 1995 ; ICC-ILEC, 1994). La diffusion de ces concepts s'est réalisée à l'occasion de conférences professionnelles et par l'intermédiaire d'ouvrages et de revues non scientifiques. Ce n'est que tardivement que le MRP et le JIT ont fait l'objet d'un intérêt croissant au niveau académique. En règle générale, ces concepts innovants conçus à l'origine par des professionnels, n'ont eu comme réponses de la part des chercheurs que des critiques qui portent sur leurs limites et leurs faiblesses. Par exemple, des articles montrent les limites du JIT (Zipkin, 1991) ou la nervousness des systèmes MRP (Blackburn et al., 1986). D'autres auteurs proposent de nouvelles approches du MRP (Trémolières, 1995) ou montrent des phénomènes d'instabilité et de chaos déterministe pouvant se produire dans des organisations fonctionnant suivant une logique JIT (Thiel, 1996a ; Takahashi et al., 1997). Des études bibliométriques sur le seul concept du JIT ont permis de classifier les travaux de recherche actuels qui portent sur les thèmes suivants : les systèmes de transport, les choix d'implantation, la logistique, les interfaces clients-fournisseurs, la qualité, la maintenance, la gestion de production et la pédagogie. Nous pouvons citer deux publications significatives portant sur le JIT ; la première en présente un état de l'art (Sohal et al., 1989) et la seconde (Groenevelt, 1993 recensant 148 publications) fait état des types de recherches sur ce concept en constatant néanmoins une insuffisance de travaux empiriques basés sur des données réelles de terrain. Quant aux limites, l'adoption du JIT nécessite de nombreuses adaptations aussi bien internes qu'externes aux firmes. Par exemple, il est nécessaire de revoir le système de comptabilisation des activités et les procédures d'évaluation du personnel qui sont directement affectés par le JIT. Vis-à-vis des partenaires externes, les fournisseurs doivent accepter de coopérer en modifiant leur structure de distribution et leurs métho- des de préparation et d'expédition des commandes. Ces changements ne pourront s'effectuer en peu de temps. Néanmoins, il est utile de noter que la majorité des implantations du JIT en entreprise a été réalisée avec succès (Groenevelt, 1993). Le concept de l'ECR étant à un stade d'adoption plus récent au niveau des professionnels, n'a pas encore reçu d' «écho » émanant des scientifiques comme les précédents concepts, le MRP et le JIT. L'ECR peut être considéré pour la distribution ce qu'était le juste à temps pour Toyota et l'industrie automobile. Il est apparu pour la première fois en 1992 lors d'une conférence du Food Marketing Institute aux États-Unis (FMI, 1993). Hammonds, alors président du FMI, a défini l'ECR comme une «stratégie qui vise à supprimer tous les coûts inutiles sur l'ensemble de la chaîne, du producteur jusqu'au client. Un coût inutile est un coût qui ne représente aucune valeur ajoutée pour le consommateur $»$. Son objectif est donc de bâtir un système réactif partant du consommateur final dans lequel distributeurs et fournisseurs travaillent en étroite collaboration pour maximiser la satisfaction du consommateur et réduire les coûts. Ce changement permet d'offrir au consommateur un meilleur service en terme de fraîcheur, de disponibilité et de qualité des produits. L'ECR synthétise également un ensemble d'idées qui circulent depuis de nombreuses années parmi les acteurs de la chaîne d'approvisionnement des produits de grande consommation. Une des idées clés est que de l'amont vers l'aval, de l'usine au magasin, le système d'approvisionnement forme un tout dont chaque maillon concoure à l'efficacité selon le principe du Just-in-Time'. L'affaiblissement d'un élément est dommageable à l'ensemble ct a contrario, la coopération entre les opérateurs d'une filière de distribution est profitable pour chacun d'eux. De nombreux pays ont su instaurer ce dialogue visant à améliorer d'une part, les relations fabricants/distributeurs et d'autre part, la réponse que ces acteurs apportent au consommateur. En ce qui concerne l'Europe qui a pris un certain retard, le comité ECR-Europe n'est en activité que depuis début 1996 (ECR Europe, 1995 ; Coopers et Lybrand 1995, 1997). Selon une étude commandée par l'European Executive ECR Board et réalisée entre mai et novembre 1995 auprès de 15 participants $(5$ distributeurs dont Promodès et 10 industriels pour 15 catégories de pro-

1. Ce concept de réapprovisionnement continu est décrit dans deux ouvrages originaux de Martin (1990, 1993). 
duits et 5 circuits de distribution), les gains potentiels pour l'Europe se chiffrent à $5,7 \%$ du prix de vente au consommateur soit environ 165 milliards de francs d'économie. Ces gains seraient issus à la fois d'une diminution des coûts opérationnels de $4,8 \%$ sur l'ensemble de la chaîne logistique et d'une réduction des stocks estimée à $42 \%$ soit 18 jours de stocks en moins pour une réduction du coût financier d'environ $0,9 \%$. Comme le montre également le graphique 1, les gains de productivité sont attendus à tous les stades de la filière.

Figure 1. Répartition des gains de productivité de l'ECR

\section{Illustration non autorisée à la diffusion}

Pour l'industrie alimentaire canadienne, le réapprovisionnement continu représenterait près de $65 \%$ de la réduction des coûts associés à la mise en œuvre d'une démarche ECR (Martin et Landvater, 1995). Citons l'exemple d'un projet pilote de réapprovisionnement continu effectué chez Oshawa Foods et H.J. Heinz du Canada qui a permis à ces entreprises de quadrupler la rotation des stocks, tout en maintenant un niveau de service de $99 \%$. (Perry et Ross, 1995). Des résultats semblables ont été obtenus en Californie où le niveau des stocks au point de vente a diminué de $61 \%$ alors que le taux de rotation des stocks est passé de 16 à 42 avec un niveau de service de $99 \%$ (Raynford, 1994).

Rivard et al. (1997) sont plus réservés quant au réel bénéfice pour le consommateur final, une fois l'intégration réalisée. Jusqu'à présent, les gains pour le consommateur se sont concrétisés sous la forme d'une augmentation du niveau de service (plus grande disponibilité ou plus grande fraîcheur). Reste également à résoudre le problème de la répartition entre les partenaires des gains apportés par l'ECR (voir discussion à ce propos dans Landry et Trudel, 1995). Ces dernières remarques et critiques nous amènent à considérer qu'une nouvelle approche comme l'ECR $s$ 'inscrit dans une problématique plus vaste qui conjugue concurrence et partenariat. Elle implique une volonté commune des concurrents/partenaires à dépasser un certain nombre d'obstacles identifiables et de définir les conditions pour que l'ECR dépasse une simple relation ponctuelle pour s'inscrire dans une démarche stratégique durable compatible avec la classique vision concurrentielle des relations verticales.

\section{Un besoin relationnel coopératif entre partenaires de la chaîne logistique}

La capacité à constituer un partenariat solide est fondamentale pour l'ECR. Des relations fiables, interdépendan- tes facilitent l'intégration de systèmes d'information efficaces en éliminant les redondances et en rationalisant les échanges. Selon la littérature professionnelle ${ }^{2}$, pour surmonter la difficulté du partage de l'information, un partenariat solide devrait être fondé sur quatre notions simples : (1) l'engagement total des directions générales des firmes partenaires, (2) un partenariat doit s'instaurer sur des bases saines où il n'a pu exister aucune friction au préalable. Il est toujours plus facile de former de vraies relations quand un haut degré de confiance lie déjà fournisseurs et commerçants, (3) un partenariat s'inscrit dans un schéma de propositions gagnant-gagnant. Pour cela, une firme doit comprendre le fonctionnement interne de ses partenaires. Les bénéfices seront d'autant mieux partagés si les deux entités connaissent leurs approches stratégiques et leurs objectifs respectifs, (4) les deux partenaires doivent $s$ 'allouer des ressources humaines, financières et techniques qui seront amenées à constituer une équipe de travail mixte performante. La finalité du partenariat définit un système commun et cohérent pour d'une part, évaluer l'efficacité globale (diminution des stocks, meilleure utilisation des moyens, meilleur rapport qualité-prix), et d'autre part, pour identifier les bénéfices (hausse du chiffre d'affaires, des marges). Tout ceci dans la seule optique de répartir équitablement tous ces avantages.

Au niveau de la recherche, Manzano (1997) souligne que « jusqu'au milieu des années quatre-vingt-dix, la notion de partenariat entre les membres d'un canal de distribution restait encore rare dans la littérature et recevait peu d'attention de la part des chercheurs en marketing (des Garets d'Ars, 1991 ; Skinner et al., 1992 ; Morgan et Hunt, 1994 ; Guibert, 1996) ». Or, certains auteurs reconnaissent que les acteurs peuvent, en coopérant, augmenter les bénéfices que chacun reçoit (Kelley et Thibaut, 1978 ; Axelrod, 1984). Skinner et al. (1992) montrent également que deux parties peuvent améliorer leur satisfaction en mettant en place une relation de coopération. Bowersox et al. (1980) notent que, comme chaque firme dépend des autres firmes du canal pour être performante, la coopération entre les membres du canal est un comportement nécessaire et vital. De ce point de vue, ces auteurs sont en accord à la fois avec Alderson qui considère la coopération comme une activité économique importante, au même titre que le conflit et que la compétition (Alderson, 1969) et avec Gill et Allerheiligen (1996) qui soulignent que les canaux ne peuvent donc fonctionner efficacement sans la coopération entre les membres du canal.

En France, d'autres économistes industriels se sont intéressés à ce besoin relationnel en terme coopératif entre partenaires de la chaîne logistique (Paché et des Garets, 1997 ; Sauvage, 1997 ; Kraff, 1998).

2. Logistiques Magazine $\mathrm{n}^{\circ} 103-104-105 ;$ LSA $\mathrm{n}^{\circ} 1423,1427,1451$, $1459,1470,1477,1480,1481 ;$ Marketing Vente $n^{\circ} 83$; Points de vente $\mathrm{n}^{\circ} 607,615,622,628,631$; Supermarket News; Traffic Management, Chain Store Age ; Décisions Marketing ; Food Business News. 


\section{La problématique de recherche}

L'évolution des marchés de plus en plus exigeants en termes de délai, de prix, de variété et de qualité des produits, explique ces évolutions paradigmatiques de l'économie industrielle et de la gestion des interfaces. L'évolution de leur organisation technique et sociale les fait basculer d'une gestion massifiée des flux vers une forme plus personnalisée produisant et distribuant en fonction du besoin des consommateurs en « juste à temps ». Mais cette transformation entraînant une réduction des stocks et misant sur la flexibilité instantanée à tout prix, rend les entreprises plus vulnérables face à des aléas internes et externes. En effet, la demande commerciale fluctuante et les dysfonctionnements internes perturbant les systèmes de production industriels, nécessitent des mécanismes de régulation efficaces. De multiples contrôles se sont mis progressivement en place pour répondre en « quasi temps réel » à ces sollicitations. Ces contrôles sont-ils réellement efficaces? Ne génèrent-ils pas des effets négatifs $a$ posteriori ? Sont-ils réellement intégrés dans un «contrôlc global» des activités économiques des filières ? Il est en effet difficile d'expliquer de manière triviale la logique de cet enchevêtrement de mécanismes complexes, moteur de la dynamique globale des échanges. Il serait imprudent et souvent faux de tenter un «réductionnisme » détaillé de ces systèmes. De nombreux praticiens se rattachent aisément à ce concept attractif qu'est le Just-in-Time dont la mise en œuvre est souvent associée de résultats impressionnants : forte diminution des stocks, réduction drastique des cycles logistiques de production-distribution... Mais en observant les comportements réels des firmes, de nombreux dysfonctionnements viennent nuire à l'application de ces différents concepts. À propos du paradigme de l'ECR, force est de constater que les articles parus dans les revues professionnelles ont tendance à apporter des réponses quasi uniques aux questions du type : Comment planifier la production des usines? Comment optimiser le chargement des camions? Comment ne pas se munir de stocks pléthoriques et comment éviter les ruptures en gondoles?... la solution étant, "à toutes ces questions, le réapprovisionnement automatique apporte des réponses afin d'optimiser l'ensemble de la chaîne d'approvisionnement ».

Pour ces différentes raisons, nous avons souhaité étudier la fragilité de ces modèles théoriques face à différentes perturbations internes et externes aux systèmes logistiques des producteurs et des distributeurs. Nous avons choisi d'étudier plus particulièrement la technique du réapprovisionnement continu (CRP Continuous Resplenishment Planning) reposant sur la communication par le distributeur des données sur les stocks et les mouvements de produits, afin de permettre aux fournisseurs de générer des propositions de commandes ${ }^{3}$.

3. Il ne s'agira pas dans ce texte de développer les techniques particulières favorisant la mise en oeuvre du concept ECR, comme par exemple l'EDI (Electronic Data Interchange), les techniques de Commande assistée par ordinateur (CAO), de lecture optique en entrepôt et en magasin, les logiciels de réapprovisionnement continu, la gestion par catégorie, etc.
Dans le paragraphe suivant, nous présentons les pratiques logistiques actuelles ainsi que les principes des techniques de tension des flux de matières et d'informations ayant pour objectif l'amélioration de l'efficience du pipeline logistique. Ensuite, nous exposons la méthodologie ayant conduit à l'élaboration de modèles génériques représentatifs de ces schémas d'approvisionnement. Une synthèse des résultats de simulations obtenus est par la suite exposée en faisant apparaître les limites des modèles proposés par différents experts sur la base de ces schémas statiques, en quelque sorte idéaux. Nous concluons cette communication par les évolutions possibles, les enjeux et certains risques de mise en œuvre de l'ECR en France.

\section{Les structures logistiques actuelles et les principes du réapprovisionnement continu}

\section{La logistique actuelle des produits frais}

En 1997, l'entrepôt est plus que jamais au cœur de la bataille commerciale que se livrent les différentes enseignes françaises. On compte en France plus de 4 millions de $\mathrm{m}^{2}$ de surfaces destinées à l'entreposage avant livraison aux GMS ce qui représente $0,36 \mathrm{~m}^{2}$ d'entreposage pour $1 \mathrm{~m}^{2}$ de surface de vente. «Avec $922000 \mathrm{~m}^{2}$, Intermarché est le plus grand logisticien de France » commente Paché (1995), chercheur à l'Université d'Aix-en-Provence. Des plates-formes situées entre les industriels et les points de vente ont permis par ailleurs de limiter les réserves en magasin. La place ainsi gagnée a pu être affectée à l'installation de nouveaux rayons de ventes. Le tableau de la figure 2 met bien en évidence la place prépondérante des entrepôts à l'heure actuelle.

En ce qui concerne les produits frais, $95 \%$ d'entre eux passent par des plates-formes chez Carrefour et $98 \%$ aux Comptoirs Modernes. Seuls Leclerc et Cora semblaient échapper à cette règle en 1996. Le rapprochement de Cora et de Carrefour a déjà fait évoluer la situation pour la plus petite de ces deux enseignes. Les livraisons directes producteurs-magasins sans passage par des entrepôts sont souvent limitées aux producteurs locaux.

À l'exception de Leclerc, la technique de l'entrepôt polyvalent gérant plusieurs familles de produits a été progressivement abandonnée. On voit apparaître aujourd'hui des entrepôts spécialisés qui permettent de stocker dans un même endroit des produits de mêmes caractéristiques. Carrefour, Prisunic et Promodès disposent ainsi de six types d'entrepôts dont certains sont exclusivement dédiés à la viande ou aux produits de la mer.

La logistique des produits frais a donc beaucoup évolué avec la généralisation des plates-formes, de plus les contraintes de DLC qu'imposent ces produits ne permettent pas la constitution de stocks spéculatifs. Les produits frais sont donc en général les premiers à bénéficier des avan- 
cées technologiques ou logistiques qui rendent plus efficace leur distribution.

Un guide des «bonnes pratiques logistiques » a été élaboré pour les denrées périssables ayant des DLC inférieures à 24 jours (ANIA et al., 1996). Il vise à appliquer la distribution en flux tendu avec pour objectif le respect des délais par la mise en œuvre d'un «compte à rebours logistique ».

Les schémas usuels rencontrés pour la distribution de denrées périssables sont présentés en figure 3.

Ce paragraphe a montré la spécificité des circuits logistiques actuels pouvant s'expliquer par des caractéristiques des circuits de distribution, des produits et de la localisation spatiale des usines/entrepôts. On comprendra que les circuits souhaitables sont ceux qui minimisent le nombre de ruptures de charge. Nous allons montrer dans le paragraphe suivant le principe du réapprovisionnement continu selon trois schémas logistiques plus simplifiés.

\section{Description des techniques et méthodes du réapprovisionnement continu}

Les conditions de réussite (largement discutées auparavant) de la gestion partagée des approvisionnements (CRP) reposent sur un partenariat étroit dans la relation commerciale et seraient les suivantes : (1) une coopération logistique qui se distingue de toutes négociations commerciales et s'intègre dans le processus d'échanges d'informations, (2) les économies sont générées d'une manière directe chez le distributeur et le fournisseur, (3) les directions générales soutiennent les deux équipes de projets, et enfin (4) un climat de confiance existe.

\section{Le principe de fonctionnement du Continuous Resplenishment Planning (CRP)}

\section{- Les pratiques actuelles}

Les deux principes émergents pour la mise en application du CRP sont (1) la planification des approvisionnements par le commerce (PAC), (2) le pilotage des approvisionnements par le foumisseur (PAF ou CRP direct) ou par le prestataire (PAP ou CRP presté).

Ne remettant pas en cause les politiques tarifaires, le premier principe (PAC) est le plus facile à mettre en application. Bien que ne permettant pas de réduction importante des coûts de stockage, il peut s'avérer être une étape intéressante avant une montée des exigences entre industriel et distributeur. Dans ce schéma, le distributeur reste maître du jeu en transmettant au fournisseur un plan d'approvisionnement sur quelques semaines. Le distributeur dispose ainsi d'un stock spéculatif virtuel (réservation et paiement immédiat mais approvisionnement au fur et à mesure des besoins réels), tandis que le fournisseur planifie plus aisément sa production. Il faut noter ici que cette technique ne concerne que des produits à DLC longues et d'autres PGC.

Dans le cas du CRP direct ou presté (second principe, PAF ou PAP), la transparence du système d'information du distributeur est totale pour le fournisseur ou le prestataire. L'approvisionnement des entrepôts du distributeur se trouve ainsi sous leur responsabilité.

Dans le cas du CRP direct, le distributeur transmet tous les jours les informations sur les sorties des entrepôts (nombre de palettes livrées aux magasins) et un état de son stock. L'approvisionneur du distributeur ne gère pas le réapprovisionnement, c'est directement l'industriel qui détermine la commande en utilisant un logiciel spécifique intégrant le niveau des stocks agréé conjointement par le distributeur, la rotation des produits des entrepôts vers les magasins et l'ensemble des contraintes logistiques ou conditions de livraisons (camions, palettes, etc.). Averti de la livraison, le distributeur confirme et valide administrativement cette opération par échanges de données informatiques (EDI). Cette commande dématérialisée correspond donc bien aux besoins de réapprovisionnements des entrepôts du distributeur et de ses magasins. Cette opération conduit souvent dans la pratique à la disparition du système plus classique des promotions basé sur les stocks 


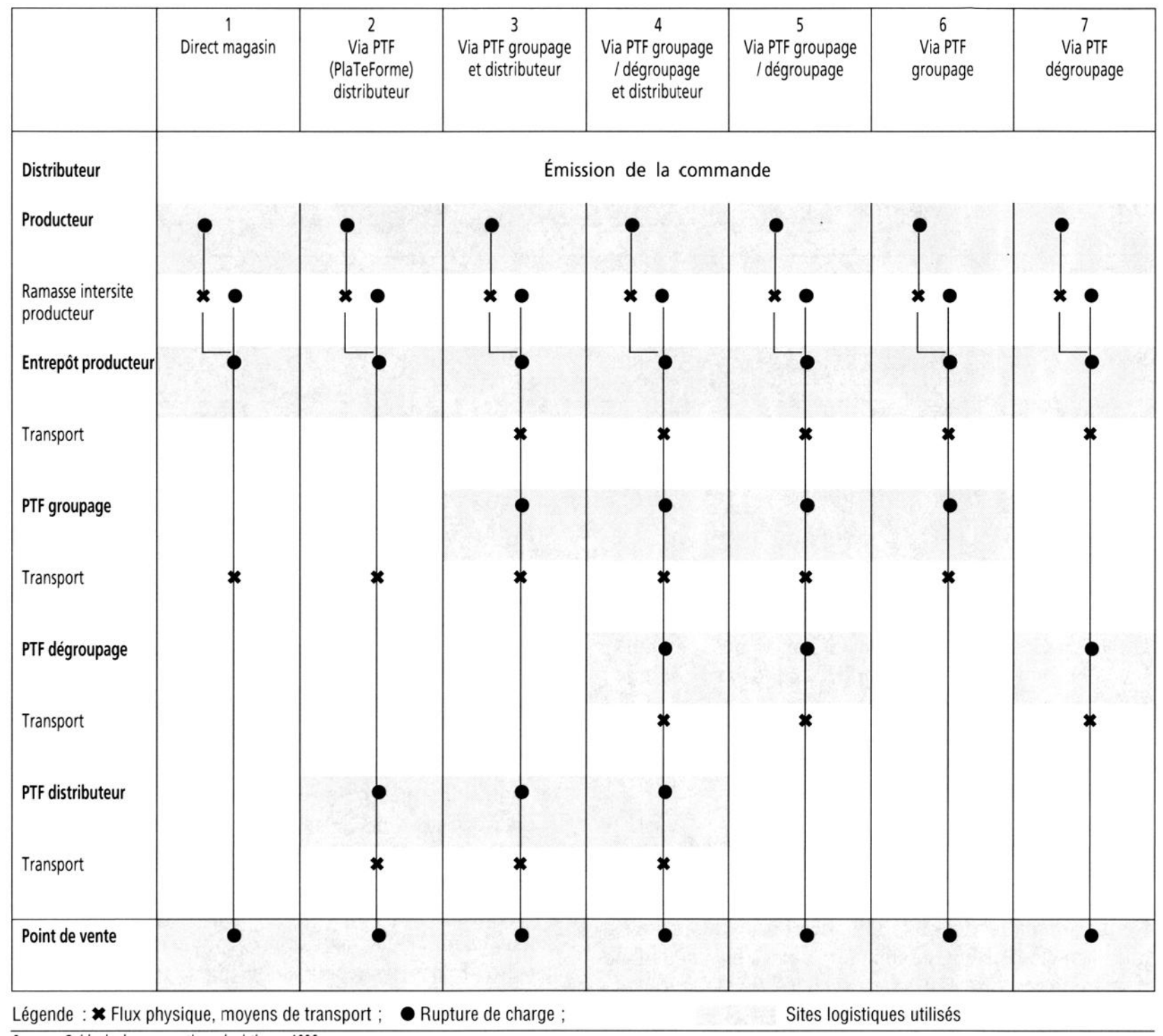

Source : Guide des bonnes pratiques logistiques, 1996.

d'opportunité du distributeur. Cette gestion aurait également tendance à favoriser les prix bas toute l'année (Every Day Low Price, EDLP).

Dans l'exemple du CRP presté, certaines expériences anglo-saxones (Wal Mart et Procter et Gamble aux ÉtatsUnis ou encore Somerfield et quelques fournisseurs en Grande-Bretagne) montrent que les industriels et les distributeurs ont fait appel à un intermédiaire chargé de passer les commandes. Ces prestataires informatiques, IBM et General Electric Informations Service (GEIS) par exemple, gèrent directement les approvisionnements des entrepôts. Dans un premier temps, ils récupèrent les données des dépôts et magasins par un réseau télématique (GEIS, IBM, Allegro, Global Network, etc.), puis ils valident ces informations et vérifient la cohérence des données en se basant sur la situation des stocks par article, sur des historiques de ventes et sur les prévisions de ventes réajustées quotidiennement. Enfin, ces prestataires envoient à l'industriel concerné une proposition de commande.

Nous présentons dans le paragraphe suivant trois schémas logistiques correspondant aux pratiques actuelles: (1) le réapprovisionnement partagé en entrepôt qui combine deux politiques, à savoir en amont, le pilotage des approvisionnements par le fournisseur (PAF) et en aval, la planification des approvisionnements par le commerce (PAC), (2) le réapprovisionnement partagé magasin, livraison entrepôt, (3) le réapprovisionnement partagé en magasin, livraison directe. Dans ces deux derniers schémas, les approvisionnements sont entièrement pilotés par le fournisseur (PAF ou CRP direct ou presté).

\section{- Les trois schémas d'organisation logistique correspondants}

Le CRP via les entrepôts n'est qu'une première étape (figure 4) avant la remontée systématique des données scanning.

Dans l'espoir d'engranger de substantiels gains de productivité, un consultant canadien explique comment les ventes déclenchent la production : «à partir du moment où la marchandise passe à la caisse, le processus s'enclenche. Toutes les heures, une procédure vérifie si le stock de chaque produit est suffisant pour le reste de la journée et le lendemain. Chaque heure, un programme planifie et anticipe les ruptures prévisibles jour après jour pour les 
deux semaines suivantes. Si l'ordinateur détecte un risque de rupture, il envoie immédiatement et automatiquement un message à la plate-forme ».

Mais on parlera réellement de réponse optimale au consommateur (cf. ECR), quand ces données proviendront directement des points de vente, comme le montrent les figures 5 et 6 .

Ces trois figures présentent donc les étapes possibles pour passer d'une logique de réapprovisionnement partagé en entrepôt (figure 4, produits à DLC longues) à une intégration totale du concept ECR grâce aux remontées en amont des informations consommateurs. Les schémas des figures 5 et 6 sont plus adaptés aux produits frais et ultrafrais bien que le schéma 1 puisse également convenir aux produits frais réfrigérés (voir par exemple Beulens, 1996).

Figure 4. Réapprovisionnement partagé en entrepôt

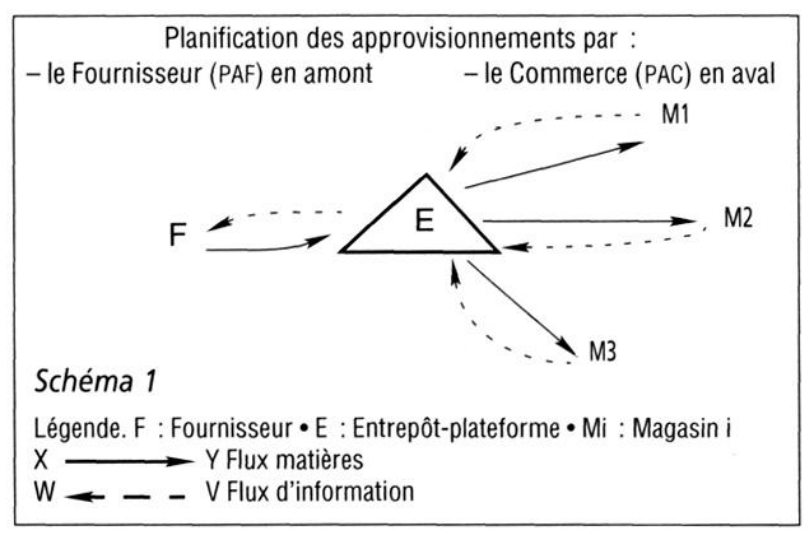

Figure 5. Réapprovisionnement partagé magasin, livraison entrepôt

Planification des approvisionnements par le Fournisseur (PAF)

Schéma 2

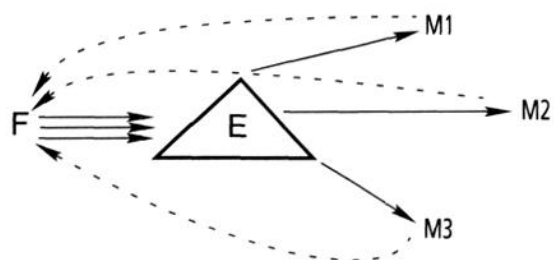

Figure 6. Réapprovisionnement partagé en magasin, livraison directe

Planification des approvisionnements par le Fournisseur (PAF)

Schéma 3

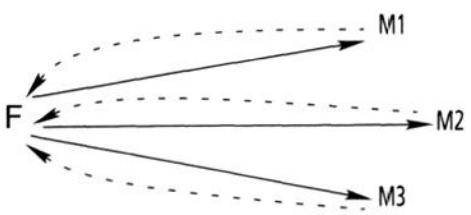

\section{Les avantages annoncés du CRP}

Toujours d'après la littérature professionnelle, la continuité des réapprovisionnements devrait apporter des avantages autant pour l'industriel que pour le distributeur et serait les suivants :
- une diminution des stocks, des ruptures, des à-coups de production ;

- une hausse du niveau de service ;

- une connaissance de la demande réelle donc des prévisions plus fiables et un meilleur planning de production ;

- une optimisation du chargement des camions-livraisons - une diminution des coûts administratifs (la fiabilité des données réduit les erreurs), de transport et de manutention; - une meilleure gestion des stocks et rotation des produits ;

- une amélioration de la fraîcheur et de la qualité des produits ;

- une élimination des tâches répétitives et sans valeur ajoutée ;

- une hausse des ventes grâce à une meilleure disponibilité des produits en magasin.

Face à ces affirmations très optimistes sur les techniques de planification et d'échanges producteur-distributeur, nous avons voulu tester l'efficacité des trois derniers schémas (figures 4 à 6) en élaborant des modèles de simulation que nous présentons dans la partie suivante.

\section{Modélisation et simulation des comportements logistiques des systèmes de réapprovisionnement continu}

\section{Proposition de modélisation}

Nous avons appuyé notre investigation sur le paradigme de la dynamique des systèmes de Forrester (1961) dont l'objectif essentiel consiste à définir les liens de causalité circulaire qui se manifestent dans un schéma cybernétique. Cette théorie se base sur le principe de l'existence de boucles fermées où une variable de contrôle agit sur une variable d'action ${ }^{4}$, qui par la suite, par l'intermédiaire d'éventuelles autres variables, contre-réagit sur cette même variable de contrôle. Ce mécanisme montre l'interaction mutuelle entre différentes variables d'un système complexe où la notion de pilotage évolue à chaque transition spatio-temporelle.

La construction progressive de ces modèles «dynamiques » passe par une phase qualitative initiale de formalisation des relations causales dans les systèmes. Il s'agit de construire la structure de feed-back par l'intermédiaire du diagramme causal. Cette approche est clairement décrite dans Forrester (op. cit.) et Roberts (1984). Richardson et Pugh (1981) proposent une approche top-down pour conceptualiser le modèle, tout d'abord en décrivant la structure physique du système, puis les flux d'information et enfin les conséquences des actions à l'origine des changements dans le système. En matière de validation des modèles, nous nous sommes inspirés a priori des trois théories existantes pour bâtir les modèles qui se sont complétés ou « ajustés » par des observations du terrain (voir

4. Au sens de Walliser (1977). 
Thiel, 1997 ; Touminet, 1997). Nous présentons dans le paragraphe suivant le principe des diagrammes causaux formalisant le fonctionnement des trois schémas logistiques présentés précédemment.

\section{Diagrammes causaux}

Les figures 7, 8 et 9 se proposent de montrer les relations causales entre les différentes variables des trois modèles génćriques de réapprovisionnement continu. En se référant aux principes de la systémique ${ }^{5}$ et en suivant une démarche constructiviste, ces diagrammes d'influence représentent les boucles de feed-back composées de variables de commande, de variables agrégées d'actions agissant sur les systèmes opérants et de délais opérationnels. Les relations de causalité sont représentées par des arcs «polarisés » par le sens algébrique de la relation. Des fonctions de transfert définissent les relations non linéaires entre différentes variables. Le type des boucles cybernétiques qui peut être positif ou négatif (boucle homéostatique), est également représenté dans les figures suivantes.

\section{Diagramme 1. Réapprovisionnement partagé en entrepôt (figure 7)}

Nous décrivons ce diagramme causal en suivant la logique de l'ECR, c'est-à-dire en partant du consommateur final (les ventes). Une première boucle de régulation (-) permet d'assurer le réapprovisionnent des linéaires et de la réserve des magasins à partir des entrepôts par un contrôle permanent des niveaux de stock. Hors promotions, ce système consiste à reconstituer les stocks en fonction des consommations réelles en magasin (cf. principe de l'Inventory Control System ICS) et ceci, dès qu'un stock minimum est atteint. Ce mécanisme est entièrement géré par le distributeur (PAC).

5. Voir par exemple Le Moigne, 1977 ; Mélèse, 1980 ; Thiel, 1993.
En ce qui concerne la gestion des approvisionnements de l'usine vers les entrepôts (seconde boucle négative (-), cf. PAF), le producteur dispose d'informations sur l'état des stocks ainsi que sur le taux de sorties des entrepôts. L'industriel planifie sa production tous les jours en effectuant des lancements dans le cas où la couverture du stock entrepôt basé sur un taux de sorties lissé, est inférieure au temps de cycle total Iproduction + transport vers l'entrepôtl. La taille du lot de production est fonction de la couverture souhaitée de stocks en entrepôt fixée par le distributeur.

\section{Diagramme 2. Réapprovisionnement partagé magasin, livraison entrepôt (figure 8)}

Dans ce cas plus adapté aux produits à DLC courtes, les commandes passées au fournisseur proviennent directement des magasins, l'entrepôt apparaît alors comme une plate-forme qui joue le rôle d'intermédiaire au niveau des flux de marchandises entre l'usine et le magasin, sans être impliqué dans les décisions. Ces opérations physiques dites d'ćclatcment à quai ou Cross-Docking assurent un flux de produits constant du fournisseur au point de vente en réduisant les stocks d'encours (de distribution) et le cycle de l'ensemble de la chaîne d'approvisionnement. Le principe est le suivant. Le fournisseur prépare les produits pour le distributeur en tenant compte des besoins des points de vente autant en terme de destination que de conditionnement. Arrivées au centre de distribution, les palettes et caisses sont lues par lecture optique. Elles sont ensuite triées et rechargées pour réexpédition sans aucune manipulation des produits et ceci dans un temps très réduit. La distribution continue via le Cross-Docking avec son corollairc la livraison allotie, optimise la productivité des stocks et de l'espace point de vente. Elle réduit les chargements massifs de produits initialement employés pour les achats spéculatifs et facilite l'expédition des produits nécessaires et strictement nécessaires au réapprovisionnement de chaque magasin.

\section{Figure 7. Diagramme causal 1}

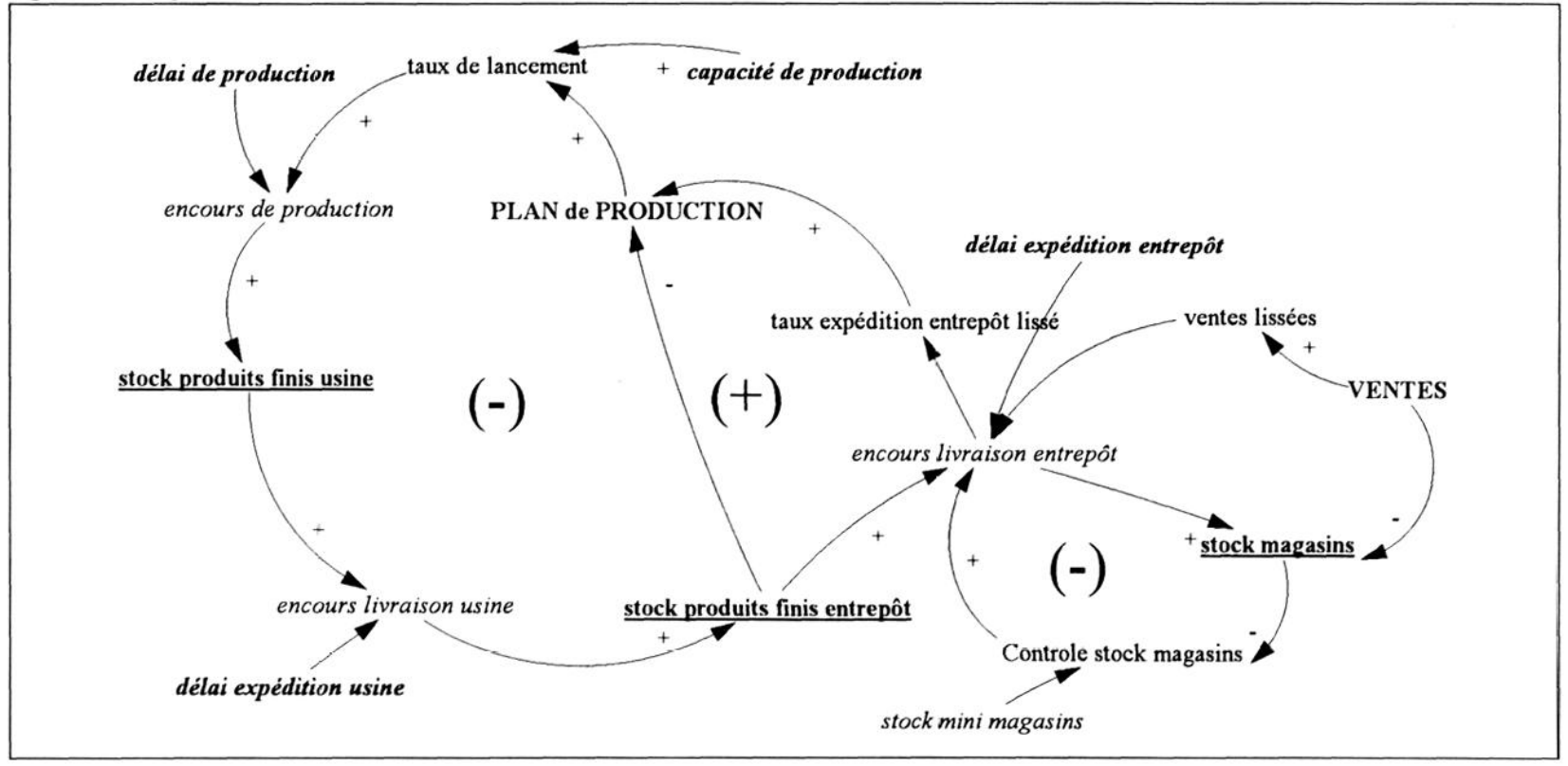




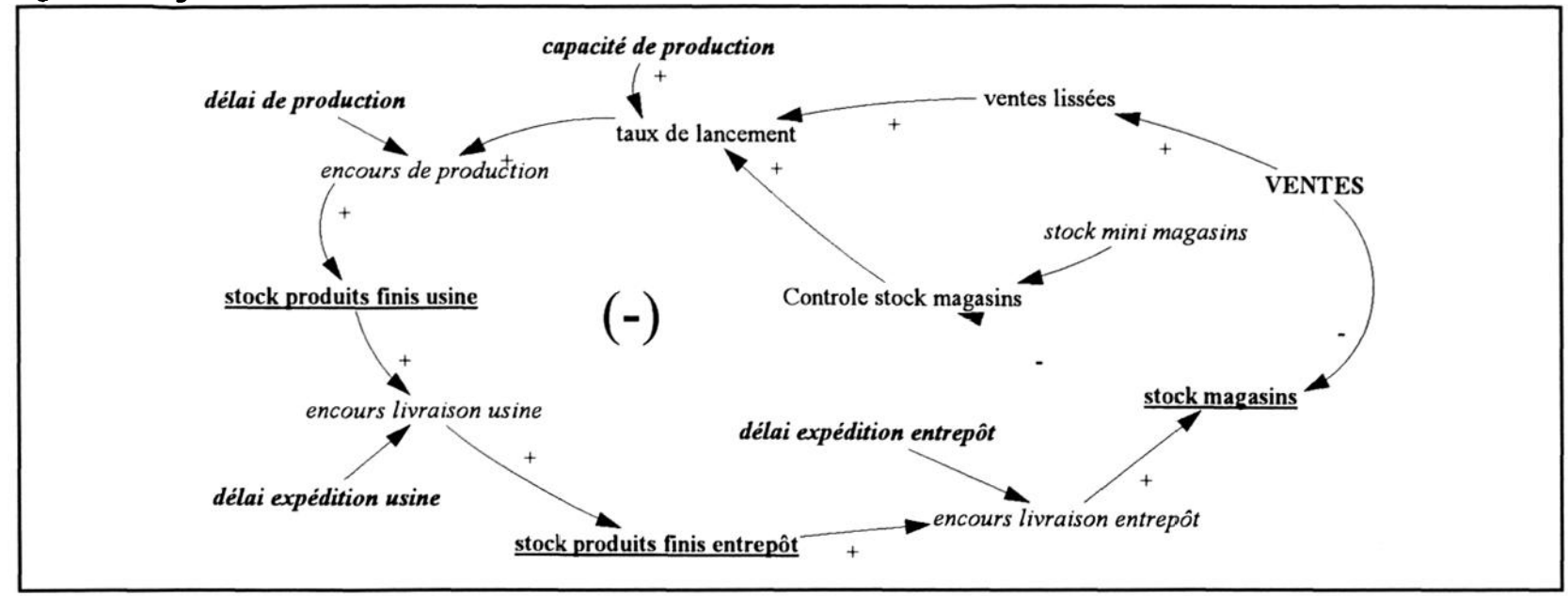

Diagramme 3. Réapprovisionnement partagé en magasin, livraison directe (figure 9)

Il s'agit ici de livraisons directes du producteur aux magasins de détail sans intermédiaires. Le fournisseur lance sa production suivant les commandes qui sont directement transmises par les magasins (comme pour le schéma précédent). Ce nouveau schéma présente l'avantage de délais courts étant donné une livraison directe. Il est actuellement peu réaliste de l'envisager systématiquement pour l'ensemble des commandes pour des raisons de coûts de transport, bien que cela se pratique fréquemment en cas de volumes importants. Précisons qu'historiquement, les usi- nes ont pendant longtemps approvisionné directement les magasins. Cette situation a très vite provoqué des goulots d'étranglement sur les quais des points de vente qui voyaient alors une multitude de camions les livrer. Les chauffeurs attendaient alors parfois des heures avant de décharger.

Pour construire les trois modèles finaux qui permettront de réaliser les simulations, nous nous sommes limités à un fournisseur (usine), trois détaillants avec et sans entrepôt/plate-forme intermédiaire (selon les schémas d'organisation des flux des figures 4,5 et 6 ). Ce modèle permet ainsi de simuler les opérations de cross-docking.

Figure 9. Diagramme causal 3

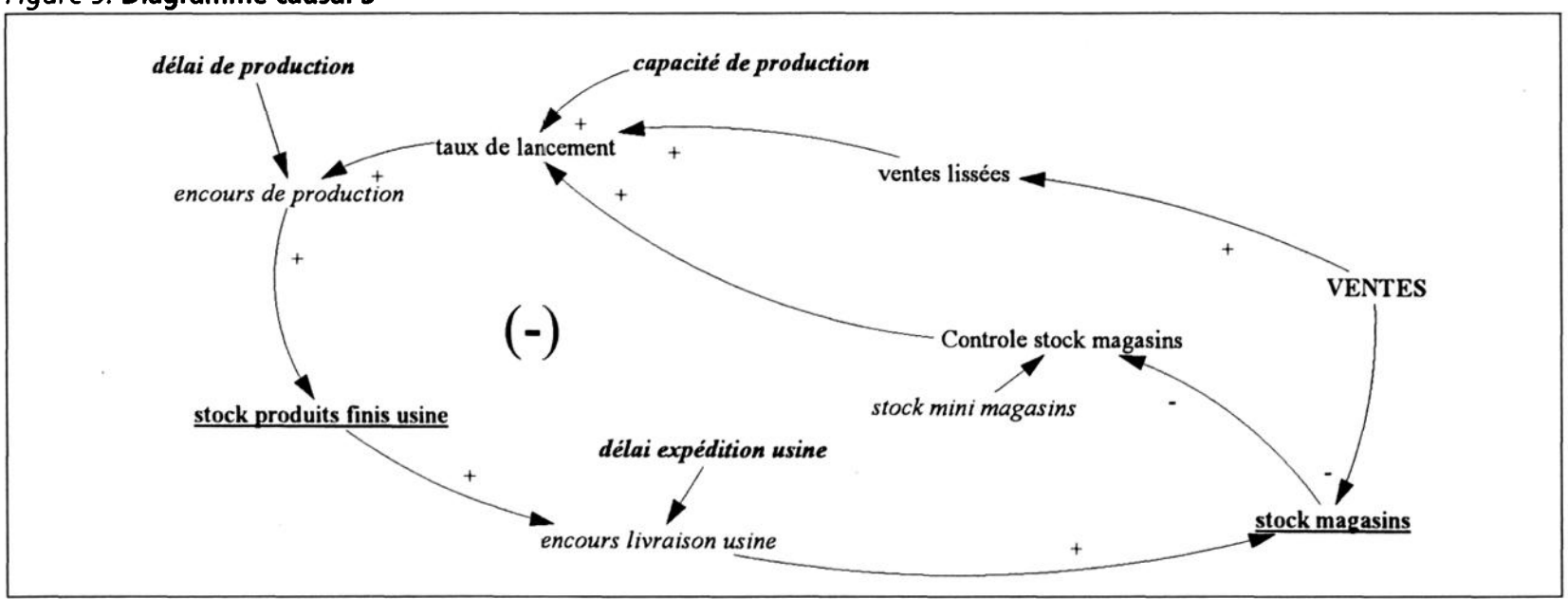

\section{Simulations des modèles}

\section{Structure des modèles génériques}

Dans la phase suivante de modélisation, Forrester (1958) préconise des modèles composés de boucles de feed-back élaborées à partir de symboles de niveaux et de taux. Les variables de niveaux décrivent l'état des systèmes par une intégration continue des résultats de l'action dans ces systèmes. Les variables de taux (ou de flux) expriment l'action par des débits d'entrée et de sortie dans les niveaux. Les niveaux existent en permanence même si toute activité cessait, alors que les taux ou flux disparaîtraient. Ce mode de représentation permet par la suite de traduire les variations de ces états par l'intermédiaire d'équations différentielles et d'en étudier leur comportement dans le temps. Nous ne présentons pas ici les modèles détaillés correspondant aux schémas logistiques précédents $^{6}$.

Cette démarche progressive de modélisation nous conduit à la simulation continue qui permet de visualiser des comportements stabilisés et/ou d'analyser des phénomènes caractéristiques de l'instabilité de certains systèmes réels sur des périodes courtes.

6. À titre indicatif, ces trois modèles sont composés respectivement de 110,139 et 117 variables de types suivants : 18,31, 24 variables de niveaux, de $31,55,42$ variables de flux, de $23,20,20$ variables auxiliaires et de $38,33,31$ constantes. 


\section{Plan d'expériences}

L'objectif des simulations a consisté d'abord à contrôler le niveau de service au consommateur (cf. manque à gagner dû à des ruptures de stocks en magasin), puis à suivre les coûts de distribution et de stockage induits par l'organisation logistique et les schémas de réapprovisionnement choisis ${ }^{7}$. Ces observations se sont effectuées toutes les heures sur une durée de 50 jours en fonction des scénarios suivants :

- Scénario 1. Évolution des ventes en magasin suivant six profils : une augmentation ou diminution brutale et durable des ventes (steps positif et négatif), des commandes exceptionnelles ou méventes très fortes sur une courte durée, mais de manière répétitive (pulses positif et négatif), des fluctuations aléatoires dans des ventes stables et des ventes avec saisonnalité. Le choix de ces scénarios peut correspondre dans la réalité à des phénomènes pouvant apparaître suite à des rumeurs diverses (par exemple une information concernant un risque de pénurie de sucre et d'huile a entraîné une hausse brutale et importante des ventes), ou des incidents sur la qualité de produits (l'exemple récent de la présence d'une seringue dans une boisson a fortement fait chuter les ventes).

- Scénario 2. Étude de la sensibilité des indicateurs de performance de la chaîne logistique à la variation de la couverture des stocks entrepôts et magasins suivant deux modes : des ventes très fluctuantes et des ventes augmentant brutalement de manière occasionnelle ou durable (pulse ou step).

Les comportements de ces systèmes seront analysés en prenant différentes hypothèses sur les variations de la demande commerciale. Les séries chronologiques provenant des ventes passées sont utiles pour tester la validité du modèle par rapport au passé connu. Mais la correspondance entre les comportements passés et les résultats du modèle ne suffit pas à assurer seule la validité du modèle et son comportement futur. Conformément aux recommandations de Forrester (1961) et de Lyneis (1988), il est donc nécessaire de tester de nouvelles hypothèses sur la demande consistant à une combinaison de cinq types d'entrées à savoir les steps positifs $-\ulcorner$ ou négatifs, les pulses positifs ou négatifs $\Lambda \Lambda \Lambda$, les bruits et les composantes saisonnières. La variation de la demande s'exprime ainsi par l'équation paramétrable suivante :

$\mathrm{D}=\mathrm{Dm} *(1+\operatorname{normal}(\mathrm{Mb}, \mathrm{Sb})+\operatorname{step}(\mathrm{Hs}, \mathrm{Ds})+$ pulse

$(\mathrm{Hp}, \mathrm{Dp}, \mathrm{Fp})+\mathrm{Am} * \cos (2 \pi \mathrm{t} / \mathrm{T}))$

avec :

$\mathrm{D}=$ demande fluctuante

$\mathrm{Dm}=$ demande moyenne

7. Étant donné le nombre de produits très limité dans nos simulations, nous suivrons l'évolution du stock moyen journalier. Attention, nos simulations ne justifierons pas une réduction des stocks obtenue par la mise en oeuvre progressive du CRP puisque nous prenons déjà l'hypothèse d'un CRP opérationnel.
$\mathrm{Mb}=$ moyenne des bruits distribués normalement $(+/ 10 \%,+/-20 \%$ et $+/-50 \%)$

$\mathrm{Sb}=$ écart-type des bruits

$\mathrm{Hs}=$ hauteur du step $(+/-10 \%,+/-20 \%$ et $+/-50 \%)$

Ds = date de début du pulse

$\mathrm{Hp}=$ hauteur du pulse $(+/-10 \%,+/-20 \%$ et $+/-50 \%$ toutes les 8 heures)

$\mathrm{Dp}=$ date de début du premier pulse

$\mathrm{Fp}=$ fréquence des pulses (toutes les 24 hcurcs)

Am = amplitude de la sinusoïde(amplitude maximale $=+20 \%,+50 \%$ et $+100 \%$ au-dessus de la moyenne)

$\mathrm{T}=$ période de la sinusoïde

Les couvertures de stock choisies sont de 0,5 jour, 1, 2 et 3 jours.

\section{Quelques résultats de simulation}

Il est utile de préciser ici que nous ne nous sommes intéressés qu'aux régimes transitoires instables, c'est-à-dire au comportement à court terme des flux logistiques suite à une perturbation dans la demande. Il est par aillcurs évident que les partenaires de cette chaîne logistique se réorganisent en cas de modification durable de leur demande commerciale.

Pour le schéma 1 (cf. figure 4), nous avons observé une instabilité du modèle et un effet de seuil en cas de commandes exceptionnelles répétitives $\Lambda \Lambda$. En effet, quand l'amplitude de ce type de commandes n'est que de $10 \%$ supérieur aux ventes «habituelles », le système logistique reste performant et assure un service parfait. Au-delà de $20 \%$, le système devient instable et les pertes de CA s'amplifient continuellement. Concrètement, cela signifie que ce schéma logistique devient risqué en cas de ventes exceptionnelles de forte amplitude.

D'autre part, en cas de baisse brutale et permanente des ventes en magasin (steps négatifs), le stock moyen en entrepôt reste à son niveau nominal quelle que soit l'amplitude de la chute de la demande. Cependant, le stock moyen en usine diminue de $17 \%$ pour une chute de $10 \%$ (cf. courbe 1 de la figure 9 ), de $25 \%$ pour une baisse de $20 \%$ (courbe 2 ) et de $63 \%$ pour un step négatif de $50 \%$ (courbe 3 ). On peut constater ici que c'est le fabricant qui réagit le plus vite à la baisse de la demande au niveau du consommateur final.

Enfin, un phénomène curieux peut s'observer en cas de baisse brutale et occasionnelle des ventes (pulses négatifs $\bigvee V V$ ). En effet, des pertes en CA sont enregistrées pour des chutes répétitives de $-10 \%$ et de $-50 \%$ par rapport aux ventes moyennes (cf. respectivement les courbes 1 et 3 de la figure 10), alors qu'en cas de diminution occasionnelle de $20 \%$, les mécanismes de régulation permettent d'éviter ces pertes (courbe 2). Cela peut s'expliquer par le degré relatif d'activation des différentes boucles homéostatiques et les phénomènes de prééminence de certaines d'entre elles. Il est connu que les systèmes déterministes peuvent être imprévisibles, du fait notamment de leur sensibilité aux conditions initiales (Lane, 1994). Une bifurcation dans un modèle continu non linéaire peut 
apparaître lors d'un changement de prééminence entre une boucle négative et une boucle positive autour d'un point d'équilibre (pour un aperçu plus détaillé de ce phénomène, on pourra se reporter à Richardson, 1984 ou Thiel, 1996b). Or, un système logistique plus «complet » que celui précédemment défini se présente comme un système structurellement complexe, composé de multiples boucles interconnectées (confère notamment Beer 1981 ; Richardson, 1992 ; Forrester, 1994). Les décisions, ou commandes d'action, sont prises en différents endroits de ce système, souvent simultanément - chaque action résultante générant de l'information, ultérieurement utilisée dans d'autres centres de décision.

Pratiquement, cette observation montre la sensibilité du schéma 1 de pilotage mixte du système logistique, à ces baisses répétitives des ventes qui pourraient s'observer sur un marché fortement concurrentiel.

Le schéma 2 (cf. figure 5) est très sensible aux ventes aléatoires, ce qui s'explique par le manque de flexibilité de la chaîne «production-logistique » face à un flux tiré en permanence. Ce même schéma assure un niveau de service acceptable en cas d'augmentation brutale de la demande jusqu'à une amplitude de $20 \%$. Au-delà de ce seuil, la capacité de production devient insuffisante.

Le schéma 3 (cf. figure 6) engendre comme prévu des coûts de distribution élevés ce qui le rend aujourd'hui assez peu réalisable. Cependant, il offre des avantages en se montrant très robuste face à une demande répétitive imprévue quelle que soit son amplitude (pulses réguliers de +10 à $+50 \%$ ). En effet, les délais de livraison étant plus courts et la réactivité meilleure, la production s'adapte donc plus facilement à l'augmentation des ventes. Par contre, ce schéma reste également sensible aux ventes aléatoires, les pertes de CA restant proportionnelles à l'amplitude des aléas de 0 à $20 \%$ environ (par rapport aux ventes moyennes), puis curieusement ce manque à gagner reste à peu près constant dans l'intervalle 20 à $50 \%$.

Figure 9. Variations des stocks

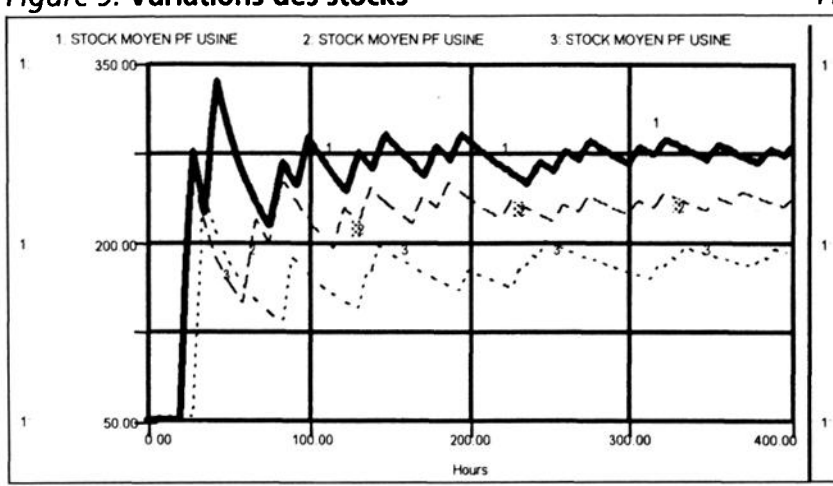

Source : Calculs de l'auteur.

\section{Synthèse des travaux et recommandations pratiques}

Par l'intermédiaire des simulations, nous avons constaté que selon différents scénarios d'évolution des ventes, ces trois schémas logistiques présentaient des instabilités et des défaillances en terme de service. Le tableau de la figure 11 montre les aspects intéressants observés en comparant ces schémas d'évolution progressive vers une logique ECR. L'utilité essentielle de ce tableau synthétique est de montrer les risques que peuvent prendre producteurs et distributeurs en choisissant un schéma de réapprovisionnement continu sans tenir compte de la spécificité du profil de la demande commerciale des produits distribués. Pour chacun de ces trois schémas (lignes du tableau), on peut observer leur efficacité en terme de service face à des profils de ventes différents (colonnes). Par exemple, pour un produit ayant des ventes à forte saisonnalité et selon son amplitude, il serait préférable de choisir les schémas 2 ou 3 plutôt que le schéma 1. Par contre, en cas d'aléas dans les ventes, le schéma 1 semble le plus robuste. Enfin, en cas d'augmentation brutale, soit permanente (colonne 1), soit répétitive (colonne 2 ) des ventes, le schéma 3 peu réaliste, serait sans doute le plus performant de par sa réactivité élevée expliquée par la suppression d'intermédiaires dans
Figure 10. Variations des pertes en CA

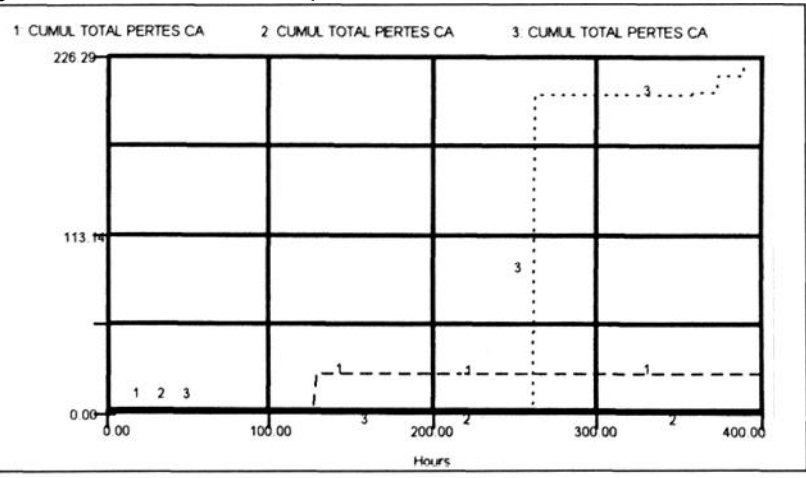

l'écoulement du flux du producteur au distributeur. Les schémas 1 sont à éviter dans le cas d'une augmentation durable des ventes, il en va de même pour le schéma 2 en cas de ventes exceptionnelles répétitives.

En ce qui concerne les produits alimentaires, le réapprovisionnement partagé en entrepôt (cf. schéma 1) présente une plus grande stabilité en cas de ventes avec de nombreux aléas, mais ne permet pas de gérer les denrées périssables sauf en cas de réfrigération. En cas de ventes fortes et imprévues, les produits frais et ultra-frais nécessitant des circuits logistiques courts (cf. schémas 2 et 3 ), seraient susceptibles de faire l'objet de ruptures en linéaires dans le cadre d'un système de réapprovisionnement partagé magasin (livraison plate-forme, cf. schéma 2). Cette dernière remarque pourrait faire l'objet d'une nouvelle réflexion sur la logistique des denrées périssables à DLV courtes et notamment en notant la robustesse du schéma 3 face à ces fluctuations, schéma malheureusement peu économique actuellement pour des petits volumes de livraison et qui pose des problèmes d'engorgement des quais de livraison des clients.

La figure 12 synthétise la sensibilité de ces schémas aux coûts de distribution. Par exemple, au niveau des coûts de 
Figure 11. Comportement des trois schémas de CRP en fonction des variations de la demande (calculs de l'auteur)

\begin{tabular}{|c|c|c|c|c|}
\hline $\begin{array}{l}\text { ANALYSE } \\
\text { DE SENSIBILITÉ } \\
\text { DE LA QUALITÉ } \\
\text { DE SERVICE } \\
\end{array}$ & $\begin{array}{l}\text { Augmentation brutale } \\
\text { et permanente des ventes }\end{array}$ & $\begin{array}{l}\text { Augmentation brutale } \\
\text { et répétitive des ventes }\end{array}$ & $\begin{array}{l}\text { Ventes très aléatoires } \\
\text { et non saisonnières }\end{array}$ & $\begin{array}{l}\text { Ventes } \\
\text { avec forte saisonnalité }\end{array}$ \\
\hline Schéma 1 & Perte croissante du CA & $\begin{array}{l}\text { Perte croissante du CA } \\
\text { dès que l'amplitude des } \\
\text { commandes exceptionnelles >+ } 20 \%\end{array}$ & Peu sensible & Très sensible \\
\hline Schéma 2 & $\begin{array}{l}\text { Perte croissante du CA } \\
\text { dès qu'augmentation brutale }>+50 \%\end{array}$ & Perte croissante du CA & Très sensible & $\begin{array}{l}\text { Sensible à partir d'un \% } \\
\text { de saisonnalité >à } 100 \%\end{array}$ \\
\hline Schéma 3 & Pas de pertes & Pas de pertes & Très sensible & $\begin{array}{l}\text { Sensible à partir d'un \% } \\
\text { de saisonnalité > à } 100 \%\end{array}$ \\
\hline
\end{tabular}

distribution, en cas de ventes périodiques, le schéma 2 a tendance à engendrer des coûts supplémentaires pour le fournisseur en cas d'amplitudes inférieures à $50 \%$, ceuxci diminuent de $25 \%$ quand l'amplitude fluctue de $+/$ $100 \%$ par rapport à la demande moyenne. Pour le schéma 3 , pour le même type de fluctuation saisonnière des ventes, les coûts de distribution baissent de $30 \%$ quand les variations autour de la moyenne passent de $50 \%$ à $20 \%$ et curieusement de $20 \%$ en passant de $50 \%$ à $100 \%$.

Enfin, la figure 13 montre la sensibilité de ces schémas aux coûts de stockage. Notons que les coûts de stockage du distributeur sont négligeables au niveau de ses platesformes pour les schémas 2 et 3 .

Figure 12. Analyse de sensibilité des coûts de distribution (calculs de l'auteur)

\begin{tabular}{|c|c|c|c|}
\hline & Augmentation brutale et répétitive des ventes & Ventes très aléatoires et non saisonnières & Ventes avec forte saisonnalité \\
\hline Schéma 1 & $\begin{array}{l}\text { Augmentation des coûts chez le fournisseur } \\
\text { de } 10 \% \text { à partir de pulses de }+50 \% \text {. }\end{array}$ & $\begin{array}{l}\text { Peu sensible chez le fournisseur } \\
\text { et chez le distributeur }\end{array}$ & $\begin{array}{l}\text { Baisse des coûts de } 15 \% \text { chez le fournisseur } \\
\text { et chez le distributeur dès que le \% de saisonnalité } \\
\text { est }>100 \% \text {. }\end{array}$ \\
\hline Schéma 2 & $\begin{array}{l}\text { Augmentation des coûts chez le fournisseur } \\
\text { de } 30 \% \text { à } 25 \% \text { pour des pulses allant } \\
\text { de }+10 \% \text { à }+50 \% \text {. }\end{array}$ & $\begin{array}{l}\text { En moyenne baisse des coûts de } 14 \% \\
\text { entre }+1-10 \text { à }+1-50 \% \text { de bruits. }\end{array}$ & $\begin{array}{l}\text { Baisse des coûts de } 5 \% \text { à } 25 \% \text { chez le fournisseur } \\
\text { quand le } \% \text { de saisonnalité varie de } 20 \% \text { à } 100 \% \text {. }\end{array}$ \\
\hline Schéma 3 & Peu sensible & $\begin{array}{l}\text { Baisse des coûts de } 15 \% \text { à } 30 \% \\
\text { entre }+1-20 \text { à }+1-50 \% \text { de bruits. }\end{array}$ & $\begin{array}{l}\text { Baisse des coûts de } 25 \% \text { dès que le } \% \\
\text { de saisonnalité est }>100 \% \text {. }\end{array}$ \\
\hline
\end{tabular}

Figure 13. Analyse de sensibilité des coûts de stockage (calculs de l'auteur)

\begin{tabular}{|c|c|c|c|}
\hline & Augmentation brutale et répétitive des ventes & Ventes très aléatoires et non saisonnières & Ventes avec forte saisonnalité \\
\hline Schéma 1 & $\begin{array}{l}\text { Augmentation du coût de stockage de } 30 \% \\
\text { chez le fournisseur en cas de pulses de }+50 \% \\
\text { et } 5 \% \text { chez le distributeur. }\end{array}$ & $\begin{array}{l}\text { insensible au niveau du fournisseur } \\
\text { et réduction de } 7 \% \text { chez le distributeur } \\
\text { en cas d'aléas de }+1-20 \% \text {. }\end{array}$ & Peu sensible \\
\hline Schéma 2 & 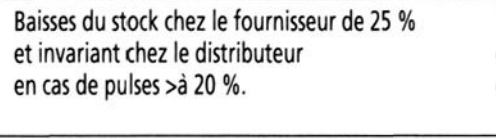 & $\begin{array}{l}\text { Réduction des stocks de } 25 \% \text { chez le fournisseur } \\
\text { et de } 15 \% \text { au niveau des points de vente } \\
\text { en cas d'aléas > à }+1-20 \% \text {. }\end{array}$ & $\begin{array}{l}\text { Peu sensible au niveau du fournisseur } \\
\text { mais augmentation du stock de } 10 \% \text { à } 30 \% \\
\text { en magasin de détail quand le \% de saisonnalité } \\
\text { varie de } 20 \% \text { à } 100 \% \text {. }\end{array}$ \\
\hline Schéma 3 & $\begin{array}{l}\text { Augmentation des stocks de } 25 \% \\
\text { chez le fournisseur dès que les pulses sont >à } 10 \% \\
\text { mais insensible au niveau des magasins de détail. }\end{array}$ & $\begin{array}{l}\text { Baisse de stocks chez le fournisseur de } 20 \% \\
\text { pour des aléas compris > à } 20 \% \text { et jusqu'à } 35 \% \\
\text { en cas d'aléas de }+1-50 \% \text {. Stocks réduits de }-25 \% \\
\text { dans les magasins entre }+-10 \text { et } 50 \% \text { de bruits. }\end{array}$ & Peu sensible \\
\hline
\end{tabular}

\section{Quelles évolutions du concept ECR en France?}

La France possède un appareil de distribution performant constitué historiquement dans une optique productiviste. Les facteurs clés sont la puissance des marques, la communication de masse, le self-service, les prix bas, la puissance d'achat, la compression des frais de personnel. Le commerce français est en effet un commerce à faible niveau d'emploi et à fort niveau de stock. Il est parfaitement régi par la loi des rendements industriels croissants et par des raisonnements d'amont en aval. C'est pourquoi Lemoine (1995) s'est interrogé sur la capacité de la
France à s'adapter à un renversement de logique par la mise en œuvre de l'ECR. En effet, lorsque l'on parle d'ECR en France, on se réfère souvent à des gains marginaux à trouver dans les fonctions administratives et logistiques. D'après une étude d'Ernst et Young, les gains engendrés par cette démarche seraient dans notre pays de l'ordre de $2,4 \%$ du prix de vente alors qu'ils sont estimés par Coopers et Lybrand à 5,7\% au niveau européen (précisons qu'aux États-Unis, l'évaluation de ces gains pour les produits de grande consommation est de l'ordre de $10 \%$ du prix de vente). La question est ainsi de savoir si l'on est prêt en France à passer d'une logique de part de marché et de productivité, à une logique de performance 
globale où l'on cherche à accroître la productivité du capital circulant. La principale variable sur laquelle il faut apprendre à jouer, c'est la variable des stocks, symptôme d'une économie poussée bien plus que tirée. Alors que la plupart des pays développés évaluent le poids de leurs stocks de 20 à $22 \%$ du PIB, le Japon qui a joué à fonds la logique du juste à temps est à $17 \%$ et la France est à près de... $30 \%$. Cette contre-performance est en quelque sorte la contrepartie de notre conception trop restreinte de la productivité.

Mais il est également utile de noter que la mise en œuvre d'un projet ECR implique de nombreuses ressources et peut entraîner d'énormes conséquences sur la performance financière de l'entreprise, de même que sur sa position concurrentielle comme le souligne Mathews (1995).
Dans cette communication, nous avons tenté de montrer à travers des simulations dynamiques que la voie vers l'Efficient Consumer Response ne se limite pas à appliquer une série de recettes d'organisation physique et à utiliser des systèmes d'information sophistiqués, mais nécessite une analyse dynamique préalable s'appuyant sur une vision globale des relations industrie-commerce. Ce qui signifie qu'une structuration fixée a priori risque de conduire à des comportements instables non maîtrisés alors qu'une modélisation préalable permettrait de représenter des phénomènes d'apprentissage et d'auto-organisation au niveau du pilotage des systèmes logistiques. Concrètement, cette analyse permettrait aux producteurs et distributeurs de mieux maîtriser leur évolution progressive vers l'ECR.

Daniel THIEL • Professeur, ENITIAA Nantes,

Groupe de recherche Agoral-gestion

\section{RÉFÉRENCES BIBLIOGRAPHIQUES}

Alderson W. Cooperation and Conflicts in Marketing Channels. In Stern L. W. (Ed.), Distribution Channels : Behavorial Dimensions, Houghton Mifflin, New York, 1969, pp. 195-210.

ANIA, ANTRODEP, CFCA, ClTI, FFOCT, FNTR, GRDP, TRANSFRIGOROUTE, UNOSTRA. Guide des bonnes pratiques logistiques : denrées périssables et autres produits alimentaires en flux tendus. 1996.

Axelrod R. The Evolution of Cooperation. Basic Books, New York, 1984.

Beer S. Brain of the Firm. Wiley,Chichester, England, $2^{\mathrm{e}}$ édition, 1981.

Beulens A.J.M. Efficient Consumer Response for fresh/refrigerated products. Internal report, Wageningen Agricultural University, 1996.

Blackburn J. D., Kropp D. H., Millen R. A. A Comparison of Strategies to Dampen Nervousness in MRP Systems. Management Science, 1986, vol. 32, n 4, pp. 413-429.

Bowersox D. J., Cooper M. B., Lambert D. M., Taylor D.A. Management in Marketing Channels. McGraw-Hill Series in Marketing, New York, 1980.

Coopers et Lybrand. Efficient Consumer Response? ECR Eurpe, Hollande, 1997.

Coopers et Lybrand. What is Efficient Consumer Response? 1995.

ECR Europe. Working Together to Fulfill Consumer wishes Better, Faster and at Less Cost. 1995, vol. 1, n 1.

Food Business News. L'ECR marque des points. 1994.

Food Business News. Le point sur l'ECR. 1995.

Food Marketing Institute, FMI. Efficient Consumer Response : getting started. 1993.

Food Marketing Institute, FMI. Update on ECR Project Priorities. ECR Alert, 1994a, $\mathrm{n}^{\circ} 7$.

Food Marketing Institute, FMI. Category Management. ECR Alert, $1994 b, n^{\circ} 9$.

Food Marketing Institute, FMI. You've probably been doing ECR but just didn't know it. ECR Alert, 1994c, n 10.

Forrester J.W. Industrial Dynamics: $A$ major breackthrough fo decision makers. Harvard Business
Review, 1958, 36, n 4, pp. 37-66.

Forrester J.W. Policies, Decisions and Information Sources of modeling. In Modeling for learning organizations, Productivité Press, System Dynamics Series, 1994.

Forrester J.W. Industrial Dynamics. Cambridge, Massachusetts, MIT Press, 1961.

Garets d'Ars (des) V. Implantation et impact des nouvelles technologies dans l'entreprise de distribution. Approche méthodologique et pratique. Thèse de Doctorat ès Sciences de Gestion, Université de Montpellier, 1991.

GENCOD EAN France. ECR réponse optimale au consommateur, une introduction. 1995.

GENCOD Informations. Perspectives et enjeux de l'ECR. 1994.

Gill L. E., Allerheiligen R. P. Co-operation in Channels of Distribution : Physical Distribution Leads the Ways. International Journal of Physical Distribution \& Logistics Management, 1996, vol. 26, n 5, pp. 49-63.

Groenevelt H. The Just in Time System. In Handbooks in OR and MS, Elsevier Science, vol. 4, 1993, pp. 387-408.

Guibert N. La relation client-fournisseur et les nouvelles technologies de l'information : le rôle des concepts de confiance et d'engagement. Thèse de Doctorat ès Sciences de Gestion, Université de Montpellier II, 1996, 284 p.

ICC-ILEC. Actes de la conférence : ECR une stratégie conjointe industrie/commerce pour une réponse optimale au consommateur. 1994.

Kelley H. H., Thibaut J. W. Interpersonal Relations : A Theory of Interdependence. New York, John Wiley \& Sons, Inc, 1978.

Kraff M. Le droit de la concurrence dans le rapport producteur-distributeur. Dans ce numéro.

Landry S., Trudel Y. Just-in-Time Supply : Cooperation, Competition and Abuse. Proceedings of the Eastern Academy of Management Sixth International Conference, Singapore, June 1995, p. 86-90.

Lane D. C. Modeling as Learning : a Consultancy Methodology for Enhancing Learning in Management Teams. In Modeling for learning organizations, Productivité 
Press, System Dynamics Series, 1994.

Lyneis J.-M. Corporate Planning and Policy Design : A System Dynamics Approach. Cambridge, Pugh-Roberts Associates, 1988.

Le Moigne J.-L. La théorie du système général. Presses Universitaires de France, 1977.

Lemoine P. Conférence sur l'ECR. Gencod, 1995.

Manzano M. Les relations coopératives entre les producteurs et les distributeurs de produits alimentaires et non alimentaires : une étude exploratoire. Working paper laboratoire GREGOR, IAE, Paris, $1997, \mathrm{n}^{\circ} 8$.

Martin A.DRP : the Gateway to True Quick Response and Continuous Replenishment. Oliver Wight Publications, 1990.

Martin A. Infopartnering : the Ultimate ECR Strategy. Oliver Wight Publications, 1993.

Martin A.J., Landvater D.D. Customer-Driven Continuous Resplenishment Program : the Ultimate ECR Strategy. 1995 Conference Proceedings, APICS, 1995, p. 260-263.

Mathews R. Spartan Pulls the Plug on VMI. Progressive Grocer, 1995, p. 64-65.

Melese J. La gestion par les systèmes - Essai de praxéologie. Editions Hommes et techniques, 1980.

Morgan R. M., Hunt S. D. The Commitment-Trust Theory of Relationship Marketing. Journal of Marketing, 1994, vol. 58, n 3 , pp. 20-38.

Paché G., des Garets V. Relations inter-organisationnelles dans les canaux de distribution : les dimensions logistiques. In Recherche et applications en marketing, 1997, vol. 12, $\mathrm{n}^{\circ} 2$, pp. 61-82.

Paché G. LSA. 1995, n 1437, p. 100.

Perry D., Ross D. Imagineering the Future of Continuous Resplenishment in the Food Industry: A Canadian Perspective 1995. Conference Proceedings, APICS, 1995, pp. 214-245.

Raynford R. A CRP Case History 1995. Progressive Grocer, 1994, pp. 44-46.

Richardson G. P. et Pugh A. L. Introduction to System Dynamics Modeling with Dynamo. Cambridge, Massachussets, the MIT Press, 1981.

Richardson G. P. Loop Polarity, Loop Dominance, and the Concept of Dominant Polarity. Proceedings of the 1984 International System Dynamics Conference, Oslo, Norway, 1984.

Richardson G. P. Feedback Thought in social Science and Systems Theory. UPP, University of Pennsylvania Press, 1992.

Rivard-Royer H., Landry S., Jobin M-H., Pasin F. Le réapprovisionnement continu : vers une gestion globale de la chaîne d'approvisionnement. Actes du colloque de l'As- sociation canadienne des sciences de l'administration, StJohn, juin 1997, pp. 1291-1304.

Roberts E. B. Managerial Applications of System Dynamics. Cambridge, Massachussets, the MrT Press, 1984.

Sandras W. A. Just In Time: Making it Happen : Unleashing the Power of Continuous Improvement. Essex Junction, VT, Oliver Wight Publications, 1989.

Sauvage T. Vers une conceptualisation de la relation d'agence entre les institutions du canal de distribution : application à la relation prestataire logistique-client. Thèse de Doctorat ès Sciences de Gestion, Université d'Aix-Marseille II, 1997.

Sekine K. Kanban : gestion de la production à stock zéro. Éditions Hommes et Techniques, 1983.

Shingo S. Maûtrise de la production - Méthode Kanban Le cas Toyota. Éditions d'Organisations, 1983.

Skinner S. J., Gassenheimer J. B. et Kelley S. W. Cooperation in Supplier-Dealer Relations. Journal of Retailing, summer, 1992, vol 68, n², pp. 174-193.

Takahashi K., Nakamura N., Izumi M. Concurrent Ordering in JIT Production System. International Journal of Operations Prod. Management, 1997, vol. 17, $\mathrm{n}^{\circ} 3$, pp. 267-290.

Thiel D. Management industriel : une approche par la simulation. Economica, 1993.

Thiel D. Unstabilities and deterministic chaos in just in time production systems. System Dynamics'96 International Conference, Cambridge, USA, 1996a, july 22-25, (soutien de la FNEGE), pp. 549-552.

Thiel D. Analysis of the behaviour of production systems using continuous simulation. International Journal of Production Research, Taylor \& Francis, 1996b, vol. 34, issue 11, p. 3227-3251.

Thiel D. Une enquête sur la flexibilité industrielle de fabricants de produits frais. Revue IAA, 1997.

Touminet G. Étude de la chaîne logistique producteursdistributeurs de produits frais. Rapport de fin d'études ENITIAA, 1997, $65 \mathrm{p}$.

Trémolières $\mathrm{R}$. Une nouvelle approche des problèmes MRP. Revue française de gestion, 1995, pp. 251-266.

Walliser B. Systèmes et modèles. Introduction critique à l'analyse des systèmes. Seuil, Paris, 1977.

Wight $O$. Production and Inventory Management in the computer age. Van Nostrand Reinhold company, 1974.

Wight O. Réussir sa gestion industrielle par la méthode MRP 2. In L'Usine nouvelle, 1984.

Zipkin P. Le mirage du just-in-time. Harvard-l'Expansion. 1991, pp. 48-57. 